\title{
Casuística de pacientes com queixa principal de alta estatura atendidos em serviço de referência em Salvador, Bahia
}

\author{
Analysis of patients with chief complaint of tall stature seen at a referral hospital \\ in Salvador, Bahia, Brazil
}

Crésio Alves ${ }^{1}$, Daniela Seabra Lima²

\section{RESUMO}

Objetivo: Descrever uma casuística de pacientes atendidos em serviço de referência em endocrinologia pediátrica com queixa principal de alta estatura.

Métodos: Revisão de prontuários de 1.980 pacientes atendidos no período de janeiro de 2003 a janeiro de 2007 , incluindo apenas os indivíduos cuja queixa principal, na primeira consulta, fosse sua alta estatura.

Resultados: Foram encontrados 16 pacientes $(0,8 \%$ do total de primeiras consultas). Destes, 12 preenchiam critérios diagnósticos de alta estatura (escore $\mathrm{Z}$ do indicador estatura para idade-ZE/I $>2$ ). Sete $(58 \%)$ eram do sexo masculino. A idade em primeira consulta variou entre 3 anos e 4 meses e 13 anos e 3 meses. O ZE/I variou de 2,18 a 5,99 e a altura-alvo, de $-1,10$ a 1,24 . Em relação às causas da alta estatura, concluiu-se que: sete pacientes ( $58 \%$ ) tinham alta estatura familiar; dois (17\%) idiopática e três (25\%) sofriam de causas patológicas (síndrome de Marfan em um e adenoma hipofisário em dois deles). Nos quatro demais, o ZE/I variou de 1,86 a 1,98, não preenchendo critérios para alta estatura. Nestes, a etiologia do crescimento excessivo foi idiopática em dois, familiar em um e causado pela síndrome de Weaver em outro.

Conclusões: A distribuição das etiologias de pacientes avaliados com a queixa de alta estatura está de acordo com a literatura, que relata ser familiar a principal causa. Embora alta estatura seja uma queixa principal pouco freqüente no consultório de endocrinologia pediátrica, a investigação deve ser cuidadosa a fim de se afastarem causas patológicas, que constituíram $25 \%$ dos casos.

Palavras-chave: estatura; transtornos do crescimento; gigantismo; pré-escolar; criança; adolescente.

\section{ABSTRACT}

Objective: To describe patients followed at a referral service in pediatric endocrinology with the chief complaint of tall stature.

Methods: Chart review of 1,980 patients evaluated from January 2003 to January 2007, being selected only individuals whose main complaint in the first consultation was tall stature.

Results: 16 patients were selected $(0.8 \%)$ and 12 of them fulfilled the diagnostic criteria for tall stature (height $\mathrm{Z}$ score for age- $\mathrm{ZH} / \mathrm{A}>2$ ). Seven (58\%) patients were males. The age at the first evaluation ranged from 3 years and 4 months to 13 years and 3 months. The $\mathrm{ZH} / \mathrm{A}$ ranged from 2.18 to 5.99 and the $\mathrm{Z}$ score for target height ranged from -1.10 to 1.24 . Regarding the causes of tall stature: seven patients $(58 \%)$ had familiar causes of tall stature, two (17\%) had idiopathic tall stature (ITS), and three $(25 \%)$ of them presented a pathologic cause (Marfan's syndrome in one and pituitary adenomas in two). In the other four patients, $\mathrm{ZH} / \mathrm{A}$ varied from 1.86 to 1.98 and did not fulfill the diagnostic criteria for tall stature. In this group, the diagnoses were: idiopathic in two, familial in one and Weaver's syndrome in another patient.

Conclusions: The distribution of etiologies of patients evaluated with the chief complaint of tall stature corresponds to the literature, in which familial cases constitute the primary cause. Although tall stature is not a frequent complaint, the investigation of these patients should be performed in a thorough way in order to early diagnose secondary causes, which constituted $25 \%$ of our study cases.
Instituição: Hospital São Rafael e Faculdade de Medicina da Universidade Federal da Bahia (UFBA), Salvador, BA, Brasil

'Doutor, professor de Pediatria, coordenador da Residência em Endocrinologia Pediátrica do Hospital Universitário Professor Edgard Santos (UPES) da Faculdade de Medicina da UFBA, Salvador, BA, Brasil

${ }^{2}$ Médica residente de Endocrinologia Pediátrica do Hospital UPES da Faculdade de Medicina da UFBA, Salvador, BA, Brasil
Endereço para correspondência:

Crésio Alves

Rua Plínio Moscoso, 222, apto. 601

CEP 40157-190 - Salvador/BA

E-mail: cresio.alves@uol.com.br

Recebido em:14/4/2008

Aprovado em: 9/7/2008 
Key-words: body height; growth disorders; gigantism; preschool; child; adolescent.

\section{Introdução}

Alta estatura é definida como altura acima de dois desviospadrão da média para sexo e idade ${ }^{(1)}$. Ela pode ser tanto uma expressão normal de fatores genéticos e familiares, como pode ter uma causa patológica. A avaliação da altura dos pais é importante na investigação da alta estatura, pois 50 a $90 \%$ da variação do crescimento é devida a fatores genéticos ${ }^{(2)}$. Crianças que crescem acima do canal de crescimento correspondente à sua altura-alvo, que tenham velocidade de crescimento excessiva ou que apresentem dismorfismos são aquelas nas quais há mais probabilidade de uma doença subjacente ${ }^{(3)}$.

Drop et $a l^{(4)}$ classificam a alta estatura como primária, secundária ou idiopática. As desordens primárias são causadas por defeitos intrínsecos nos ossos ou no tecido conjuntivo. Muitas vezes são de origem genética, podendo estar associadas a anomalias cromossômicas, desordens monogênicas ou síndromes de causa desconhecida. Nesses casos, a alta estatura geralmente tem início no período prénatal, caracterizando-se por crescimento desproporcional e presença de dismorfismos. Exemplos de causas primárias de alta estatura são as síndromes de Klinefelter, X Frágil, Beckwith-Wiedemann, Weaver, Sotos, Marfan e Beals. As causas secundárias são, em geral, de origem hormonal, ocorridas por mudanças na velocidade de crescimento. No grupo das idiopáticas, estão incluídas a alta estatura constitucional e a familiar, que representam as causas mais comuns de alta estatura ${ }^{(5)}$.

A procura ao atendimento médico para investigação de alta estatura não é muito freqüente na prática clinica diária ${ }^{(6)}$. Tal fato pode estar associado ao conceito de que o crescimento acima da média é um bom indicador de nutrição, bem-estar social e qualidades positivas ${ }^{(4)}$. Por outro lado, é freqüente o impacto negativo da alta estatura na vida de alguns pacientes que apresentam como conseqüência uma dificuldade de relacionamento e vestuário, adoção de postura cifótica e isolamento social, por se sentirem diferentes da maioria ${ }^{(2,4,5)}$. O diagnóstico etiológico precoce é importante já que há a possibilidade de terapias hormonais e ortopédicas e, ainda, intervenções cirúrgicas no caso de etiologia tumoral ${ }^{(5)}$.

Nesse contexto, o objetivo do presente estudo foi descrever uma casuística de pacientes atendidos num serviço de refe- rência em endocrinologia pediátrica cuja queixa principal, por ocasião da primeira consulta, fosse alta estatura.

\section{Métodos}

Estudo retrospectivo descritivo analítico, realizado por meio da revisão do livro de registros de atendimento, selecionando apenas pacientes cuja queixa principal, motivadora do primeiro atendimento, tenha sido a alta estatura. Este trabalho foi realizado no Hospital São Rafael, em Salvador, Bahia, hospital-escola com Residência em Pediatria e conveniado com a Faculdade de Medicina da Universidade Federal da Bahia (UFBA) para colaborar com o treinamento da Residência em Endocrinologia Pediátrica. O Hospital São Rafael é uma unidade de referência em endocrinologia pediátrica, que atende pacientes de todo o estado e conta com cinco ambulatórios semanais, unidades de internamento, inclusive Unidade de Terapia Intensiva, serviço de emergência, centro cirúrgico e serviços de apoio diagnóstico. Além de assistência médica, o Hospital São Rafael desenvolve atividades de ensino e pesquisa na área de endocrinologia pediátrica sob a coordenação de dois professores universitários da UFBA. O levantamento de dados compreendeu o período de janeiro de 2003 a junho de 2007, no qual foram atendidos 1.980 novos pacientes. Destes, foram identificados 16 pacientes cuja queixa principal, motivadora do primeiro atendimento, foi a alta estatura.

A confirmação do diagnóstico de alta estatura foi realizada naqueles indivíduos que apresentassem o escore $Z$ do indicador estatura para idade (ZE/I) superior a 2. Foram avaliadas as seguintes variáveis: idade e altura à primeira consulta, gênero, desenvolvimento puberal, presença de dismorfismos, idade óssea, altura dos pais e diagnóstico final. As estaturas dos pacientes, de seus pais e a altura-alvo foram convertidas em escore ZE/I para permitir melhor comparação entre os indivíduos. A avaliação diagnóstica complementar, por ser um estudo de revisão de prontuários, foi realizada de modo individual, a depender das informações clínicas, laboratoriais e de imagem apresentadas por cada paciente.

Mesmo não sendo necessária a obtenção do termo de consentimento livre e esclarecido, os dados clínicos e laboratoriais foram mantidos em sigilo de acordo com os pressupostos que regulamentam a ética em pesquisa. O estudo foi aprovado pelo Comitê de Ética em Pesquisa do Hospital São Rafael. 


\section{Resultados}

Dos 16 pacientes $(0,8 \%$ de um total de 1.980 novas consultas), identificados como tendo a queixa principal de alta estatura por ocasião do seu primeiro atendimento, 12 (75\%) preenchiam critérios diagnósticos para esta condição ( $\mathrm{ZE} / \mathrm{I}>2)$. Destes, sete (58\%) eram do sexo masculino. A idade à primeira consulta variou de três anos e quatro meses a 13 anos e três meses. O escore ZE/I médio para altura foi 3,19 (variação: 2,13 a 5,99) e da altura-alvo 0,24 (variação: $-1,10$ a 1,24). O estadiamento puberal (critérios de Tanner) descrito em 11 (92\%) pacientes, mostrou três pré-púberes e oito púberes. A idade óssea (método de Greulich \& Pyle) estava avançada um ano em relação à idade cronológica em oito pacientes e igual a esta em quatro (dois: alta estatura familiar, um: adenoma hipofisário e um: síndrome de Marfan). Apenas um paciente com síndrome de Marfan relatou história familiar de alta estatura (pai e irmão acometidos pelo mesmo problema). A Tabela 1 sumariza os dados referentes à idade, gênero, altura, altura-alvo, desenvolvimento puberal, idade óssea, e diagnóstico desses 12 pacientes. Em relação aos sintomas associados (vide categorização numérica dos pacientes na Tabela 1) observou-se que: o paciente “\#1", com adenoma hipofisário, apresentava galactorréia e cefaléia, o “\#6", também com adenoma hipofisário, tinha sintomas de acromegalia e o paciente “\#9”, com síndrome de Marfan, apresentava ectopia de cristalino e aracnodactilia. A tomografia computadorizada de crânio foi realizada no paciente \#1, evidenciando processo expansivo selar (adenoma hipofisário). A ressonância magnética de hipófise-hipotálamo foi realizada no paciente \#6, revelando um macroadenoma hipofisário. O Quadro 1 descreve as principais informações relativas aos pacientes com etiologia neoplásica para a alta estatura. O ecocardiograma no paciente com diagnóstico clínico de síndrome de Marfan foi normal. A dosagem do fator de crescimento insuliniforme tipo 1 (IGF-1) foi obtida em 4 indivíduos (pacientes \#2, 7, 8 e 12), sendo alterada no paciente \#2, com alta estatura familiar. A proteína carreadora do fator de crescimento insuliniforme (IGFBP-3) foi testada em três indivíduos (pacientes \#3, 8 e 12), sendo elevada no paciente \#12 com alta estatura idiopática. O diagnóstico final das causas de alta estatura ( $\mathrm{ZE} / \mathrm{I}>2)$ mostrou que sete pacientes $(58 \%)$ tinham alta estatura familiar; dois (17\%), alta estatura idiopática e três (25\%) causas patológicas: um com síndrome de Marfan e dois adenoma hipofisário.

Nos outros quatro pacientes com queixa principal de alta estatura, mas que não preenchiam critério diagnóstico a condição, a ZE/I variou entre 1,86 e 1,98. A etiologia do crescimento excessivo foi idiopática em dois casos, familiar em um caso e síndrome de Weaver em outro. A Tabela 2 mostra

Tabela 1 - Gênero, idade cronológica, idade óssea, escore Z estatura/idade (ZE/I), altura-alvo, estadiamento puberal e diagnóstico final dos pacientes com diagnóstico confirmado de alta estatura

\begin{tabular}{|c|c|c|c|c|c|c|c|}
\hline $\mathbf{P}$ & G & IC & 10 & Altura/cm (ZE/I) & AA/cm (escore Z) & Tanner & Diagnóstico \\
\hline 1 & $\mathrm{~F}$ & $4 a$ & $4 a$ & $130,0(5,99)$ & $156,0(-1,1)$ & M1 P3 & $\mathrm{AH}$ \\
\hline 2 & $\mathrm{M}$ & $3 a 4 m$ & $4 a 6 m$ & $108,0(2,45)$ & $185,0(1,24)$ & $\mathrm{P} 1 \mathrm{G} 1$ & $\mathrm{AEF}$ \\
\hline 3 & $\mathrm{~F}$ & $10 a$ & $11 a$ & $157,0(2,73)$ & $172,0(-0,94)$ & M3 P2 & AEF \\
\hline 4 & $\mathrm{~F}$ & $11 \mathrm{a} 6 \mathrm{~m}$ & $13 a$ & $165,5(2,38)$ & $171,0(0,36)$ & M5 P5 & AEF \\
\hline 5 & $M$ & $12 \mathrm{a} 10 \mathrm{~m}$ & $15 a$ & $177,5(2,80)$ & ND & ND & AEF \\
\hline 6 & $M$ & $12 a 3 m$ & $14 a$ & $185,0(4,25)$ & ND & P2 G2 & $\mathrm{AH}$ \\
\hline 7 & $\mathrm{M}$ & $12 a 3 m$ & $13 a 6 m$ & $181,0(3,77)$ & $182,0(0,67)$ & P3G3 & AEI \\
\hline 8 & $\mathrm{~F}$ & $12 \mathrm{a} 7 \mathrm{~m}$ & $13 a 6 m$ & $170,5(2,18)$ & $171,5(1,13)$ & M5P5 & AEF \\
\hline 9 & $M$ & $13 a 3 m$ & $14 a$ & $180,5(2,78)$ & ND & P4G4 & SM \\
\hline 10 & $M$ & $13 a$ & $14 a 6 m$ & $180,5(3,01)$ & $181,0(0,60)$ & P5G4 & AEF \\
\hline 11 & $\mathrm{~F}$ & $5 a 2 m$ & $7 a 10 m$ & $126,0(3,21)$ & $166,0(0,44)$ & M1P1 & AEF \\
\hline 12 & $M$ & $7 a 6 m$ & $9 a$ & $142,0(2,77)$ & $175,0(-0,16)$ & P1G1 & AEI \\
\hline
\end{tabular}

P: paciente; G: gênero; F: feminino; M: masculino; IC: idade cronológica (em anos-a e meses-m); IO: idade óssea (em anos-a e meses-m); AA: altura-alvo; ND: não documentado; Tanner: estadiamento puberal; AH: adenoma hipofisário; AEF: alta estatura familiar; AEI: alta estatura idiopática; SM: síndrome de Marfan. 
Quadro 1 - Resumo dos pacientes com alta estatura de etiologia neoplásica.

\begin{tabular}{|c|}
\hline Paciente \#1 \\
\hline Sexo feminino, 4 anos \\
\hline Motivo da consulta: alta estatura \\
\hline Sintomas associados: galactorréia, cefaléia \\
\hline $\begin{array}{l}\text { Altura da mãe: } 155 \mathrm{~cm} \text {; altura do pai: } 170 \mathrm{~cm} \text {; altura-alvo: } \\
156 \pm 5 \mathrm{~cm}\end{array}$ \\
\hline $\begin{array}{l}\text { Altura: } 130 \text { (>p 99); ZE/l: 5,99; peso: 40,5kg (>p 99); } \\
\text { Tanner: M1/P3 }\end{array}$ \\
\hline Avaliação hormonal: GH: 539ng/dL (<10); cortisol: \\
\hline 17,2mcg/dL (5-25); estradiol: 5,0 pg/mL (<30); \\
\hline $\mathrm{FSH}:<0,1 \mathrm{mlU} / \mathrm{mL}(0,1-2,8) ; \mathrm{LH}: 0,12 \mathrm{mlU} / \mathrm{mL}$ \\
\hline (0,1-1,6); prolactina: $25,8 \mathrm{ng} / \mathrm{dL}(0,2-20) ; \mathrm{TSH}: 1,4 \mathrm{uUI} / \mathrm{mL}$ \\
\hline $\begin{array}{l}(1,1-1,8) ; \mathrm{T} 4 \mathrm{~L}: 0,7 \mathrm{ng} / \mathrm{dL}(1-5,5) \\
\text { Idade óssea: } 4 \text { anos }\end{array}$ \\
\hline TC de crânio: processo expansivo selar \\
\hline Conduta: ressecção cirúrgica \\
\hline Diagnóstico final: adenoma hipofisário \\
\hline Paciente \#6 \\
\hline Sexo masculino, 12 anos e 3 meses \\
\hline Motivo da consulta: alta estatura \\
\hline Sintomas associados: acromegalia \\
\hline Altura dos pais: não disponível \\
\hline Altura: 185 (>p 99); ZE/l: 4,25; peso: 78I \\
\hline Tanner: P2/G2 \\
\hline $\begin{array}{l}\text { Avaliação hormonal: GH (menor valor após teste de } \\
\text { sobrecarga com glicose): } 21,8 \mathrm{ng} / \mathrm{dL} \text {; cortisol: } 11 \mathrm{mcg} / \mathrm{dL} \\
\text { (5-20); prolactina: } 22,3 \mathrm{ng} / \mathrm{dL}(0,2-20) ; \mathrm{T} 4 \mathrm{~L}: 1 \mathrm{ng} / \mathrm{dL}(1,1- \\
\text { 1,8); TSH: } 1,64 \mathrm{uUl} / \mathrm{mL}(1-5,5)\end{array}$ \\
\hline Idade óssea: 14 anos \\
\hline RNM: processo expansivo selar \\
\hline Conduta: ressecção cirúrgica \\
\hline Diagnóstico final: adenoma hipofisário \\
\hline
\end{tabular}

GH: hormônio do crescimento; ZE/I: escore Z do indicador estatura para idade; FSH: hormônio folículo estimulante; LH: hormônio luteinizante; TSH: tireotrofina; T4L: tiroxina livre; TC: tomografia computadorizada; RNM: ressonância nuclear magnética. os principais dados clínicos, antropométricos, de desenvolvimento ósseo e puberal e diagnóstico final desses pacientes.

\section{Discussão}

A alta estatura definida pelo critério antropométrico de $Z E / I>2$, apesar de ser uma queixa principal pouco freqüente nos ambulatórios de endocrinologia pediátrica ( $0,8 \%$ nesta casuística), é um distúrbio a ser investigado sistematicamente.

Devido a uma maior aceitação social da alta estatura constitucional e familiar, tem ocorrido redução do número de pacientes avaliados para esta condição. Por isso, o número de publicações, muito freqüentes nas décadas de 1960 e 1970, diminuiu consideravelmente a partir de então(7). Além disso, observa-se um aumento da altura média das pessoas, o que contribui para uma melhor aceitação da "alta estatura”. Em 1865, a altura média dos recrutas alemães era de $165 \mathrm{~cm}$, em 1965 era de $178 \mathrm{~cm}$ e, em 1980, passou para $182 \mathrm{~cm}^{(2)}$. Outro fato interessante é que altura final esperada para que se inicie uma terapia com estrógenos, em meninas com alta estatura, mudou de $175 \mathrm{~cm}$ em 1956, para $183 \mathrm{~cm}$ em 1978 e $188 \mathrm{~cm}$ em 1999 ${ }^{(7,8)}$. Em direção oposta a isso, encontra-se o número cada vez maior de indicações para o uso do hormônio de crescimento, até mesmo em crianças com baixa estatura idiopática, para torná-los adultos mais altos. A principal diferença entre esses grupos de pacientes é o gênero: enquanto consultas para avaliação de alta estatura são mais comuns em meninas, os meninos são os que mais procuram auxilio médico para crescer. Nesta casuística, a distribuição por gêneros foi exatamente igual $(50 \%$ para cada). Embora mais meninos (58\%) tivessem o diagnóstico de alta estatura confirmado, uma proporção maior de meninas (75\%) fez parte do grupo no qual este diagnóstico, apesar

Tabela 2 - Gênero, idade, escore $Z$ de altura e altura-alvo e diagnóstico final dos pacientes que embora tivessem queixa principal de alta estatura, não preencheram critérios diagnósticos para esta condição

\begin{tabular}{ccccccc}
\hline $\mathbf{P}$ & $\mathbf{G}$ & IC & IO & Altura/cm (escore Z) & AA/cm (escore Z) & Diagnóstico \\
\hline 3 & F & $14 a 6 m$ & $15 a$ & $172,0(1,98)$ & $170,5(1,37)$ & AEF \\
9 & F & 1 a 8m & $3 a$ & $89,0(1,90)$ & $162,5(-0,09)$ & AEI \\
13 & M & $15 a 7 m$ & $14 a 6 m$ & $186,0(1,86)$ & $168,0(1,38)$ & S. de Weaver \\
14 & F & $6 a 4 m$ & $6 a 10 m$ & $128,0(1,91)$ & $165,0(0,28)$ & AEI \\
\hline
\end{tabular}

P: paciente; G: gênero; F: feminino; M: masculino; IC: idade cronológica (em anos-a e meses-m); IO: idade óssea (em anos-a e meses-m); AA: altura-alvo; AEI: alta estatura idiopática; AEF: alta estatura familiar. 


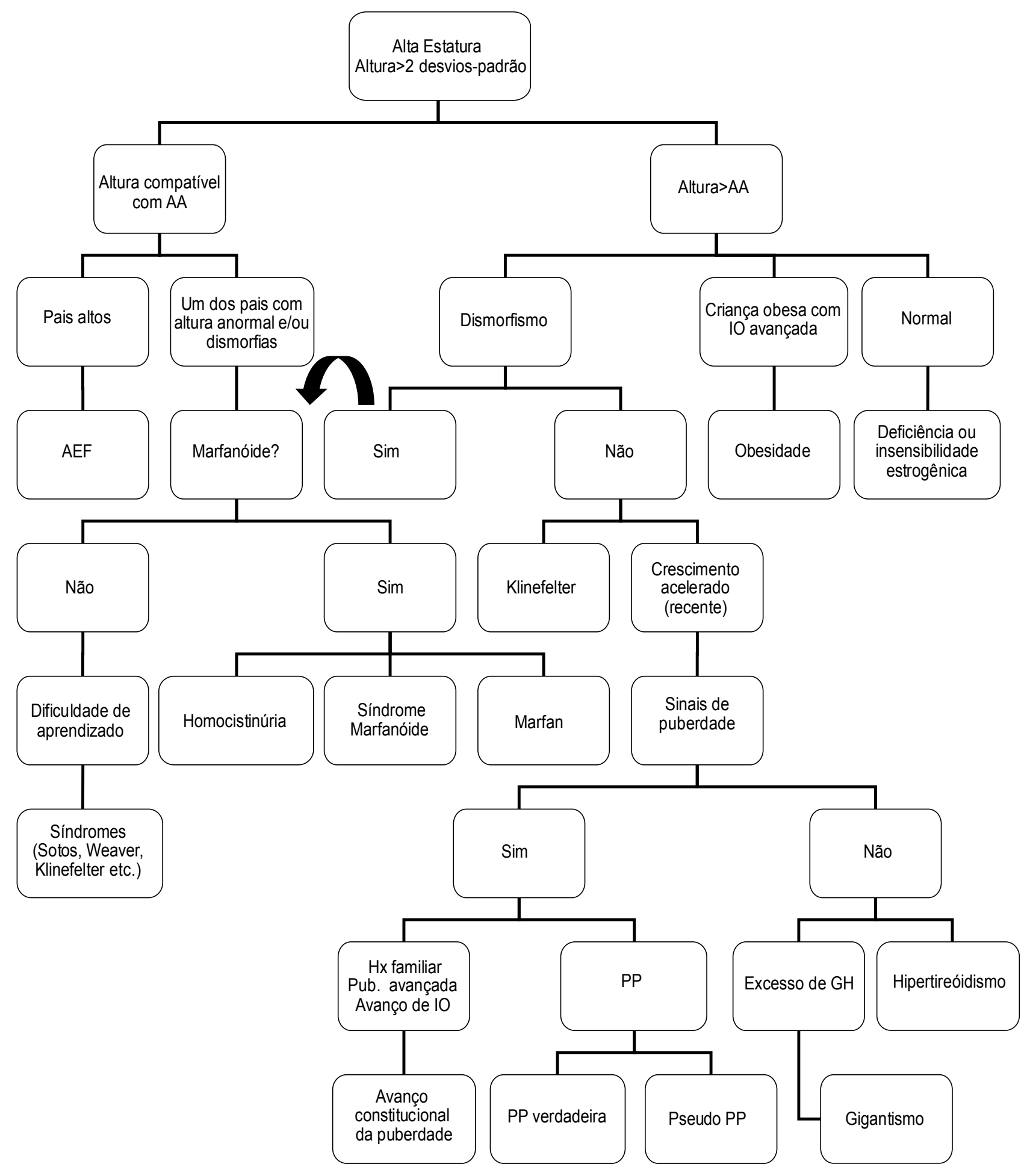

AA: altura-alvo; AEF: alta estatura familiar; IO: idade óssea; Hx: história; Pub: puberdade; PP: puberdade precoce; $\mathrm{GH}$ : hormônio do crescimento.

Figura 1 - Fluxograma para investigação de alta estatura (modificado de Drop et al(4)). 
de relatado na queixa principal, não foi estabelecido, o que está de acordo com literatura: há uma maior preocupação dos pais em relação ao crescimento excessivo quando os pacientes são do sexo feminino.

Dos pacientes queixosos de alta estatura, dois tiveram diagnóstico de síndrome genética: um com Marfan e outro com Weaver. Porém, apenas um, com síndrome de Marfan, preenchia o critério de alta estatura; no paciente com síndrome de Weaver a ZE/I foi 1,86. A síndrome de Marfan, com prevalência de 1:5.000, é causada por um distúrbio do tecido conjuntivo de herança autossômica dominante, resultado de uma mutação no gene da fibrilina (FBN1), no cromossomo $15^{(3,9)}$. O paciente com Marfan da série estudada teve sua suspeita e confirmação diagnóstica facilitada pelo fato de o pai e um dos irmãos também serem portadores. $\mathrm{O}$ diagnóstico precoce da síndrome de Marfan é importante para que mortes prematuras devido à dilatação e dissecção da aorta possam ser prevenidas. A síndrome de Weaver é um distúrbio raro, de etiologia desconhecida cuja maioria dos casos é esporádica. Ela se caracteriza por excesso ponderal, crescimento exagerado, camptodactilia, dismorfismos faciais e retardo puberal ${ }^{(6)}$.

$\mathrm{Na}$ infância, os adenomas secretores de hormônio do crescimento respondem por 5 a $15 \%$ dos adenomas pituitários, podendo causar gigantismo ${ }^{(10)}$. No grupo estudado, dois pacientes apresentaram este diagnóstico: uma menina de quatro anos com hiperprolactinemia, galactorréia e cefaléia e um menino de 12 anos e três meses com acromegalia.

Inquérito com endocrinologistas da Lawson-Wilkins Pediatric Endocrinology Society, em 1977, mostrou que 50\% dos endocrinologistas usavam medicamentos para tratar alta estatura naquela época, em contraste com $23 \%$ em $1999^{(7)}$. Nenhum dos pacientes precisou de terapia hormonal para diminuir a previsão de altura final. Estudos mostram que as indicações de tratamento da alta estatura são restritas, podendo ser utilizados hormônios sexuais em altas doses, visando o fechamento prematuro das epífises, ou o uso desses hormônios em baixas doses para induzir a puberdade precocemente ${ }^{(2,4)}$. Lee e Howell ${ }^{(7)}$ discutem o emprego de terapia estrogênica em meninas com alta estatura, mostrando que os critérios para se iniciar um tratamento vêm sendo alterados constantemente e o uso de medicações, cada vez menos freqüente. Desse modo, os artigos mais recentes relatam principalmente pacientes com alta estatura patológica, com ênfase em seu diagnóstico e tratamento ${ }^{(11)}$.

Este estudo teve algumas limitações associadas ao seu delineamento. Por ser uma revisão de prontuários, é suscetível a uma não-uniformidade das investigações diagnósticas, o que reduz seu poder analítico, não podendo atribuir significado clínico que permita comparações, uma vez que nem todos pacientes foram submetidos à mesma avaliação; isso permite apenas uma análise descritiva dos dados. Por ter tido o objetivo de avaliar somente pacientes queixosos de alta estatura por ocasião da primeira consulta, os dados aqui apresentados não nos permitem concluir que as causas da alta estatura sejam representativas do universo de pacientes nos quais este diagnóstico é realizado independentemente de sua queixa principal. Entretanto, este trabalho chama atenção para o problema e ressalta a ênfase clínica merecida em sua investigação. A presente casuística também serve de subsídio para o planejamento de futuros estudos que objetivem um aprofundamento maior na avaliação da investigação, etiologia e co-morbidades da alta estatura. Levando-se em consideração os fatos supracitados, esta casuística mostra ser familiar a principal causa de alta estatura; percebeu-se, ainda, que o tratamento dessa condição não é tão requisitado pelos pais e/ou paciente, ou mesmo prescrito pelos médicos, como no passado $^{(6)}$. Além disso, o estudo mostra que a investigação deve ser cuidadosa a fim de se afastarem as causas patológicas, nesta série presente em $25 \%$ dos casos $^{(12)}$. O fluxograma da Figura 1 sugere critérios de orientação para a investigação sistemática da alta estatura.

Diante das variadas causas de alta estatura, uma cuidadosa investigação diagnóstica realizada de modo sistemático faz-se necessária, uma vez que, em se tratando de distúrbios endocrinológicos, pode haver tratamento clínico; em se tratando de síndromes genéticas há a possibilidade de aconselhamento genético e identificação precoce de possíveis neoplasias associadas e, nos casos de etiologia tumoral, há possibilidade de tratamento cirúrgico. 


\section{Referências bibliográficas}

1. Kochi C, Longui CA. Critérios de avaliação do crescimento normal. In: Monte O, Longui CA, Calliari LE, Kochi C, editors. Endocrinologia para o pediatra. 3. ed. São Paulo: Atheneu; 2006. p. 31-6.

2. Drop SL, De Waal WJ, De Muinck Keizer-Schrama SM. Sex steroid treatment of constitutionally tall stature. Endocr Rev 1998;19:540-58.

3. Summers KM, West JA, Peterson MM, Stark D, McGill JJ, West MJ. Challenges in the diagnosis of Marfan syndrome. Med J Aust 2006;184:627-31.

4. Drop SL, Greggio N, Cappa M, Bernasconi S. Current concepts in tall stature and overgrowth syndromes. J Pediatr Endocrinol Metab 2001;14 (Suppl 2):975-84.

5. Kant SG, Wit JM, Breuning MH. Genetic analysis of tall stature. Horm Res 2005;64:149-56.

6. Toralles MB. Crescimento excessivo. In: Monte O, Longui CA, Calliari LE, Kochi C, editors. Endocrinologia para o pediatra. $3^{\mathrm{a}}$ ed. São Paulo: Atheneu; 2006. p. 85-91.
7. Lee JM, Howell JD. Tall girls: the social shaping of a medical therapy. Arch Pediatr Adolesc Med 2006;160:1035-9.

8. Barnard ND, Scilalli AR, Bobela S. The current use of estrogens for growth-suppressant therapy in adolescent girls. J Pediatr Adolesc Gynecol 2002;15:23-6.

9. Alpert BS. Tall stature. Pediatr Rev 1998;19:303-5.

10. Colao A, Pivonello R, Di Somma C, Tauchmanova L, Savastano S, Lombardi G. Growth hormone excess with onset in adolescence: clinical appearance and long-term treatment outcome. Clin Endocrinol (Oxf) 2007;66:714-22.

11. Bouthelier RG, Lapunzina P. Follow-up and risk of tumors in overgrowth syndromes. J Pediatr Endocrinol Metab 2005;18 (Suppl 1):1227-35.

12. Zeferino AM, Filho AA, Bettiol H, Barbieri MA. Acompanhamento do crescimento. J Pediatr (Rio J) 2003;79 (Suppl 1):S23-32. 